\title{
HISTOPLASMOSE CUTÄNEO-MUCOSA, RELATO DE DOIS CASOS EM IRMÃOS
}

\author{
Raymundo Martins Castro *, Helena Muraco **, Evandro A. Rivitti * \\ e Petronio José Affonso Filho *:**
}

\begin{abstract}
A comunicaçâo versa sobre dois casos de histoplasmose cutâneo mucosa ocorrendo em dois irmãos. No primeiro doente as lesões cicatrizaram sob terapêtica especifica recidivando porém três anos após. ao nivel do sistema nervoso central. Ersas lesôes levaram a doente ao óbito. No segundo dcente o diarjnóstico foi estabelecido mais precisa e precocemente e sob terapêtica especifico houve remissäo completa das lesões, estando o doente sob observação. Em ambos os casos havia grande semelhança do quadro clinico com o da blastomicose sul-americana (paracoccidiodomicose). No primeiro doente o diagnóstico foi estabelecido à necropsia e no segundo em vida, pelo encontro do Histoplasma capsulatum $\mathrm{em}$ cortes histológicos e pelo isolamento do fungo por cultura.
\end{abstract}

A histoplasmose não é micose profunda de observaçăc muito frequente no Brasil. Lacaz \& col. (7) em revisão feita $€ \mathrm{~m} 1955$, registraram até então 10 casos publicados. Armond \& col. (3) em investigacão sobre a. histcplasmose infecção em Minas Gerais, ccletaram mais 11 casos na literatura nacicnal. A esses há a acrescentar os 14 cascs humanos e um canino observados por Paula (8) em epidemia no Estado do Rio de Janeiro e os oito de observação de Fava Netto \& ccl. (6) em surto ocorrito no litoral norte de Estado de São Paulo.

Le todos esses casos somente um, observado por Almeida \& Lacaz $(1,2)$ e os dois relatados por Becker \& Guimarães (4) apresentaram lesões cutâneas e/ou muco- sas. Nos casos de Becker \& Guimarães a comprovação diagnóstica foi somente histopatológica.

Tendo tido oportunidade de estudar dois casos de histoplasmose com lesões cutâneomuccsas julgamos conveniente publicar as referidas observaçôes.

\section{CASO 1}

N. A. B., feminina, de seis anos, natural e procedente de Caitité na Bahia, veio à ccnsulta em 17-3-1360 porque há um uno e meio ccmeçou a apresentar amolecimento dos dentes. Logo após surgiram edema e ulceração do lábio superior.

Departamento de Medicina Tropical e Dermatolouip (Prof. J. A. Meira), - Divisão de Dermatologla (Prof. S. A Prado Sampaiol da Faculdade de Medicina da Universidade de Säo Paulo.

* Proiessor docente.

** Médico assistente.

*** Médico residente

Recebido para publicaçăo em 4.5.72. 


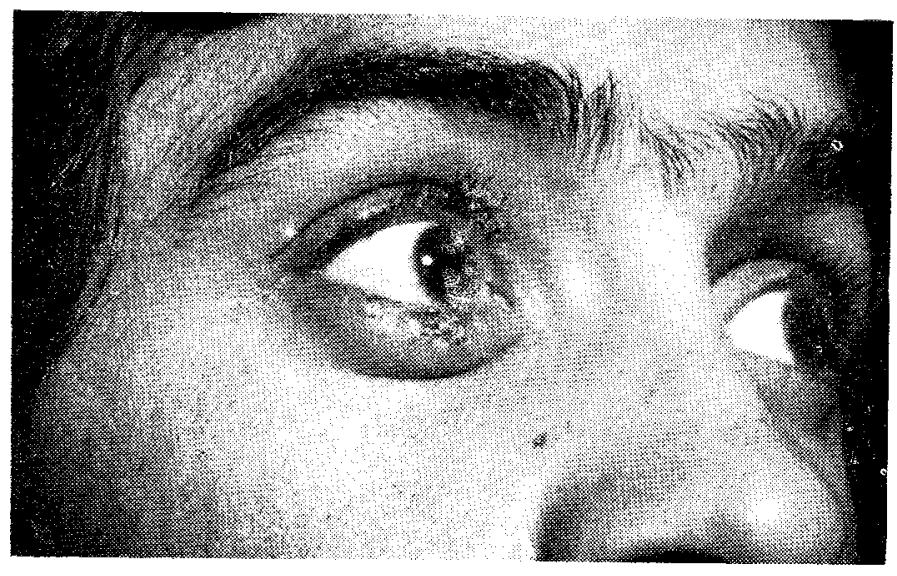

Fig. 1 - Caso 2, Lesão palpebral

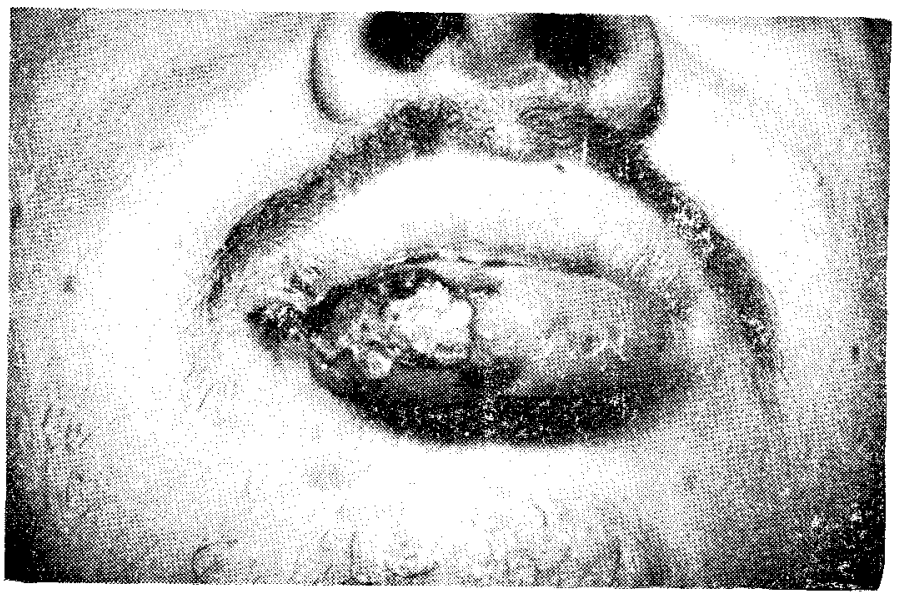

Fig. 2 - Casc 2. Lesão labial 
Foi então tratada com numerosos medicamentos entre os quais sulfamídicos (sulfametoxipiridazina). Obteve melhora com cicatrizaçãc quase total em um mês de tratamento. Abandonado o tratamento, rápidamente houve recidiva com amolecimento dos dentes, edema e ulcerações nos lábios havendo também lesão cutânea ulcerosa no mento. Há cinco dias não conseguia deglutii.

Referia que um irmão havia tido mal semelhante há três anos porém com remissāc rápida.

Ao exame havia edema e lesõ $s$ ulcerosas nos lábios e região mentoniana com certa microstomia decorrente de cicatrizaçãc.

As gengivas estavam edemaciadas e os cientes amolecidos. A microstomia limita a abertura da bcca o que impedia o exame do cro-faringe. C restante do exame físico não mestrava nada de anormal.

Internada com diagnóstico provisório de biastomiccse foi feita a investigação etiológica, duas biopsias que mostraram processo granulomatoso, mas não foram encontrados parasitos em laminas coradas por hematoxilina-ecsina e Gomori e prcvas soroićgicas (reaçōes de fixação do complementc e de precipitação) para blastomicose resultaram negativos.

Nos demais exames de laboratóric feitos hematológicc, bilirrubinas, hemosedimentaçãc, sorologia de sifilis, proteinas e fraçōes, uréia, urina tipo 1 e reação de $\mathrm{Ma-}$ chadc-Guerreiro nada foi encontrado de ancrmal. A radiografia do tórax näc mcstrcu tanbém qualquer anormalidade.

C quadro elínico era de tal forma su gestivo de blastomicose oue fci instituido tratamento por anfotericina $\mathrm{B}$ com rápida melhcre de dcente. Recebeu $700 \mathrm{mg}$ dc antibióticc com o alie houve ccmpleta cicatrizaçāc das lesóes sendo dada alta com a dcente em bcm estado em 31-5-1960. Voltcu à consulta somente em 9-11-1863, agora ccm nove anos. Referia a mãe oue passara bem até janeiro de 1963 época na qual começu a perder cabeio. Dois meses depois teve crise convulsiva ficando 12 horas inccnsciente. $\mathrm{C}$ quadro convulsivo repetiu-se ccm menor intensidade cito dias depois. Hé vinte dias vinha apresentando cefaléia intensa com náuseas e vômitos.
O exame físico mostrava doente em bom estado geral, afebril, puiso de 100 e PA $10 \mathrm{x}$ 7. Apresentava lesão ulcerosa recoberta por crosta no couro cabeludo, região do vertex. As Iesōes referidas na internação anterior permaneciam cicatrizadas. $O$ restante do exame mostrava comprometimento do sistema nervoso central. A doente mostrava-se confusa não obedecendo às ordens, não conseguia manter-se em posição ostostática apresentando sinais de irritaçāo meningea (discreta rigidez de nuca e sinais de Kernig e Brudzinski positivos). Teve evclução tumultuosa com vômitos em jato, febre, contraturas musculares, crises convulsivas, crises de apnéia, coma e morte em 11-12-1963.

Lcs exames subsidiários realizados é de interesse relatar o resultado do exame liquórico (18.11.63): era límpido, incolor $\mathrm{ccm} 77$ celulas por $\mathrm{mm} 3$ das quais $70 \%$ eram linfocitos, $10 \%$ eram monocitos $\mathrm{e}$ $10 \%$ granulccitos neutrofilos; as proteínas totais $46 \mathrm{mg}$, os cloretos $670 \mathrm{mg}$ e a glicose $38 \mathrm{mg} 100 \mathrm{ml}$. A reação de Takata-Ara foi positiva $(+)$ bem como as de Pandy e Ncnne. As reações imunolćgicas para sífilis e cisticercose foram negativas sendo-o também as reações de fixação de complementc e precipitação para blastomiccse. $O$ €xame micológico e a pesquisa de b.a.a.r. no liquor fcram negativos (exame microscópicc e cultura). Nesta segunda internaçãc, sem diagnóstico preciso mas com qua$\mathrm{drc}$ clínico e liquórico sugestivos de neurotubercuicse foi a doente tratada com sulfiscrascl, estreptomicina, hidrazida e PAS.

A necropis foi encontrada meningoercefalite por Hiscriasma capsulatum não havendo sido evcontrada histoplasmose em nenhum cutro órgāo.

\section{CASC \&}

E. B. D. masculino de 18 anos, natural e proeadentc de Caitité na Bohia, veio a conslita em 11.6.1971 puroue tinha ferida na boca há três meses. Contava que ha trés meses teve "bolha" na borhecha direita quo se ulcercu, havendc progressão da lesão que se estendeu à comissura labial direita, gengivas, mentc e fossa nasal esauerda. Há três meses também dor no globo cuular direitc e pálpebras, surgindo logo a seguir edema no local. 


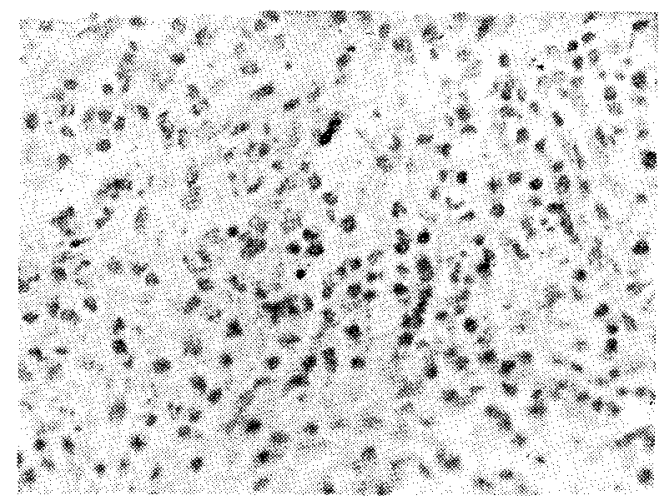

Fig. 3 - Histopiasma capsulatum em corte histo. lógico de pele. Caso 2. Coloraçăo pelo métcdo de Gomori (prata-urotropina).

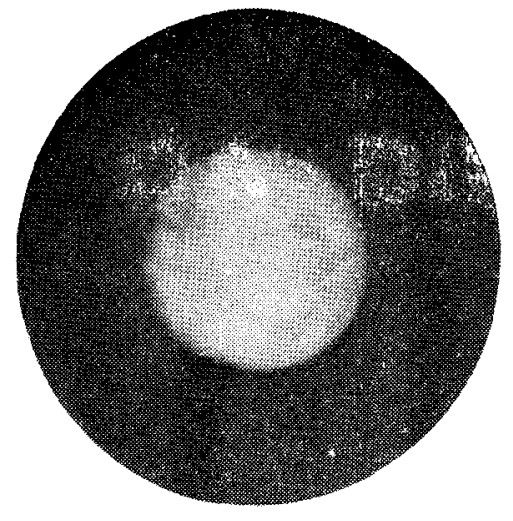

Fig. 4 - Cultura de Histopiasma capsulatum isolada do caso 2.

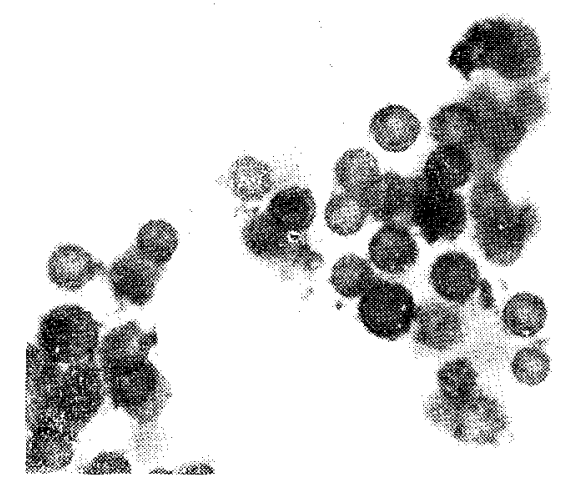

Fig. 5 - Microfoto da cultura. Estalagmosporos caracteristicos. 
Ao exame encontrava-se lesão vegetante, exulcerada de fundo finamente granulose, nc lábio inferior, comissura labial direita e região bucinatriz direita. Na conjuntiva palpebral inferior direita há exulceração circular de cerca de $3 \mathrm{~mm}$., a fossa nasal esquerda está eritematosa e parcialmente obstruida por crosta hemática. No mento há lesão ulcerosa recoberta por crosta hemática (figs. 1 e 2 ).

Nos antecedentes relatou que aos très anos teve "micose" no lábic não sabendo o tratamento que foi feito. Informou também que uma irmã teve doença semelhante havendo falecido de meningite. Informou também que trabalhava em feira, estando há seis anos ausente da zona rural onde nascera.

Internado com diagnóstico provisório de blastomicose, a investigação paa diagnóstico etiológico foi dirigida nesse sentido.

Dois exames micológicos diretos foram negativos bem como duas bicpsias que mcstraram processo granulomatosc com pesquisa de parasitas negativa. As reaçōes sorclćgicas para blastomicose resultaram num título 3,5 para a fixação do ccmplemento e prova do precipitinas negativa Face esses resultados foi instituido tratamento com sulfadimetoxina, na dose de 3,0 g diárias, o aue promoveu gradativa melhora do doente. Uma terceira biopsia de pele mostrou a presenca de parasitos com mcrfclogia de H. capsulatum (fig. 3). Com esse elemento foram procedidas culturas de material das lesōes em meio de Sabouraud, culturas essas que resultaram positivas para Hiroplosma capsulatum ligs. 4 e 5). Dos demais exames feitos há de inte resse referir que a prova da histoplasmina foi negativa e que o exame radiológic do tórax foi normal.

\section{COMENTT́RIOS}

Julgamos de interesse registrar estas duas observações pelas razões que vão expostas a seguir.

1) A histoplasmose-doenȩa ainda é pouco registrada na nossa literatura, sendo de interesse publicar novas observaç̃es.
2) Conforme vimos no inicio, de todos os casos publicados no Brasil somente três apresentavam lesōes cutâneas e/ou mucosas. Com relação às formas cutâneo-mucosas da histoplasmose é interessante referir que nossa primeira observação dá apoio à idéía de Moore \& Jorstad (8) e Curtis \& Grekin (5) que afirmam serem esses casos freqüentemente formas iniciais de histoplasmose generalizada.

3) Nossos dois doentes precediam do estado da Bahia, unidade de Federação onde já foi registrado outro caso (7) e de onde também foi isolado o H. capsulatum do solo em região endêmica de calazar $(10)$. A semelhança clínica que pcde haver entre essas duas reticulo-endoteliooses parasitárias e também a semelhança entre os agentes etiológicos faz com que tenhamos que ser cuidadosos na diagnose diferencial em doentes dessa procedência. Nossos doentes iiustram também a dificuldade que pode haver com relaçāo à diagnose diferencial com a paracoccidiodomicose. As lesôes de nossos doentes eram clinicamente indistinguíveis das da paraccccidiodomicose e mesmo o enccntro do $H$. capsulatum em cortes histclógicos pode não ser elemento diagnóstico de total segurança pois as formas "minor" do $P$. braoiliensis podem ser confundias com o $H$. carsulatum. E pois leccmendável, para estabelecer-se diagnóstico crreto, tentar-se isolar o fungo das lesões, c que foi cbtido com facilidade do nosso segundo doente.

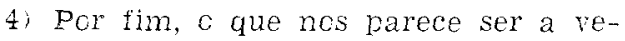
rificação mais interessante em nossas observaçós, a ccorrência em dois irmãos de "histcplasmcse-doença". Nāo encontramos registro semelhantes na literatura nacional. Fava Netto \& col. (6) estudando a cccrência familiar da paracoccidiodomicose anaisam as scguintes hipóteses para $\mathrm{ex}-$ plicaı a ocorrência familiar de "micose-docnça": deficiencis de ordem genética quanto à resistência à infecção; ínte de infeccác, com a aual membros da mosma familia teriam maici probabilidadc de cntrar en contato; moléstia contagiosa c. como tal, podendo atingir com maior frequiência membros da mesma família: importância da virulência do microbio c da carga infectante no caso de so tratal da mesma fonte de infcccão ou de muléstia contagiosa. 
Com relação à histoplasmose, micose na qual após os exaustivos estudos feitos nos Ectades Unidos, ficcu cabalmente demonstrado aue o desenvolvimento da histcplasmose-doença é uma raridade nos indivî- duos infestados. É pois sugestiva a hipótese de deficiência de ordem genética para justificar a ocorrência de "micosı-doença" em irmãcs que entraram em contato com a. mesma fonte de infecção.

\section{SUMMARY}

Two cases of muco-cutaneous histoplasmosis in brothers are presented The first case under specific treatment improved quickly but had recidive with losicns in the nervous system, three years afferwords, leading to death.

In the second case, the aiagnosis was performod earlier ana under oecifis treatment clinical cure curred. The rrtient is still under clinionl control.

In both cases, the clinical aspects of muco-cutrneous histoslo mo is were cimilar to those of SGuth. Amerisan blastomycosis (paracoccialicacmycosis).

The firts diagnosis was post-mortem and the second one was by fungus isolation, in culture, and the presence of the Histoplasma capsulatum in the nistological sections.

\section{REFERENCIAS BIBLIOGFÁFICAS}

1 - ATMEIDA, F. de \& LACAZ, C. S. Cogumelc do gênero Histcplasma isolado de lesões de cromcmicose. Asscciação de fungos nas lesões. Folia Clinica et Biologica, 9: 65-69, 1839.

2 - ALMEIDA, F. de \& LACAZ, C. S. Consideracōes em tornc de duas amostras de Histoplasma isolada de dermatite verrucosa e de escarro. Angis da Faculdade de Medicina da Universidade de Sãc Pauic 17: $561-$ 57.1241.

3 - ARMONL, S. FURTADO, T. A. OLI VEIRA, L. G. O. \& NEVES, J. - Investigacões scbre a histoplasmose infeccão em Minas Gerais. Revista da Associação Médica de Minas Gerais, 21: $27-32 \quad 1070$.

4 - BECKER, F. F. L. \& GUIMARÃES, S A. C. Histoplasmose citomicose reticulo endotelial. Estudo histopatclogica de dois casos. Hospital (Rio de Janeiro! 63: 1125-1133, 1963.

5 - CURTIS, A. C. \& GREKIN J. N. Histoplasmasis. A raview of the cutaneous and adjacent mucous membrane manifestations with a repcrt of three cases J. A. M. A. 134: 1217, 1847.
6 - FAVA NETTO, C CASTRC, $P$. FADILHA-GONCALVES, A. \& DILICN, N. I. -- Coorrência familiar da blastomicose sulamericana. A proposito de 14 casos. Rev. Ints. Med. Trop. S. Paulo, 7: 332-336, 1965.

5 - LACAZ, C. S.; LUISI, A. DEL NEGRC, G. \& CASTRO, R. M. - Histopiasmose na infância. Comentários scbre um novo caso. Revisão da literatura nacional. Noves dados sobre a histcplasmina $e m$ nosso meio. Revis-te Paulista de Medicina, 47: $495-509$. 1956 .

\& - MOORE, M. \& JCRSTAD, L. H. Histoplasmosis and its importance to Ctorhinclaringologists. A review with a report of a new case. Annals Orolcgy, Rhinology and Laringology, 52: $77 \mathrm{si}, 1943$.

\% - PAUla, A. de - Microepidemia d histcrlasmose. Revista Servico Nocional de Tuberculose, 3: 11-20, 1958.

10 - SILVA, M. E. - Isolamento do Histowlrems crpsulatum do sclo, em zona endêmica de calazar, na Bahia Bcletim da Fundaçāo Gonçalo Muniz. 9: $1-8,1956$. 